\title{
Positive solutions for a singular fractional nonlocal boundary value problem
}

\author{
Luyao Zhang ${ }^{1}$, Zhongmin Sun² and Xinan Hao ${ }^{1 *}$
}

\section{"Correspondence:}

haoxinan2004@163.com

'School of Mathematical Sciences,

Qufu Normal University, Qufu,

P.R. China

Full list of author information is

available at the end of the article

\section{Springer}

\begin{abstract}
We investigate a singular fractional differential equation with an infinite-point fractional boundary condition, where the nonlinearity $f(t, x)$ may be singular at $x=0$, and $g(t)$ may also have singularities at $t=0$ or $t=1$. We establish the existence of positive solutions using the fixed point index theory in cones.
\end{abstract}

MSC: 26A33; 34B08; 34B10; 34B16; 34B18

Keywords: Positive solution; Singular; Infinite-point fractional boundary condition; Fixed point index

\section{Introduction}

We consider the existence of positive solutions for the following fractional nonlocal boundary value problem:

$$
\begin{cases}D_{0^{+}}^{\alpha} x(t)+\lambda g(t) f(t, x(t))=0, & t \in(0,1), \\ x(0)=x^{\prime}(0)=\cdots=x^{(n-2)}(0)=0, & D_{0^{+}}^{\beta} x(1)=\sum_{i=1}^{\infty} \alpha_{i} D_{0^{+}}^{\gamma} x\left(\xi_{i}\right),\end{cases}
$$

where $\lambda>0$ is a parameter, $D_{0^{+}}^{\alpha}, D_{0^{+}}^{\beta}$, and $D_{0^{+}}^{\gamma}$ denote the Riemann-Liouville fractional derivatives, $2 \leq n-1<\alpha \leq n, 1 \leq \beta \leq n-2,0 \leq \gamma \leq \beta, \alpha_{i} \geq 0,0<\xi_{1}<\xi_{2}<\cdots<\xi_{i-1}<\xi_{i}<$ $\cdots<1(i=1,2, \ldots)$, and $\Gamma(\alpha-\gamma)>\Gamma(\alpha-\beta) \sum_{i=1}^{\infty} \alpha_{i} \xi_{i}^{\alpha-\gamma-1}$. The function $f(t, x)$ may have singularity at $x=0$, and $g(t)$ may be singular at $t=0$ and/or $t=1$.

Fractional differential equations describe many phenomena in various fields of science and engineering [1-4]. For the development of the fractional differential equations, see [523 ] and the references therein. Recently, the existence of positive solutions for fractional differential equation multipoint boundary value problems (BVPs) have been studied by many authors; see [24-33]. Using the compression expansion fixed point theorem due to Krasnosel'skii, Henderson and Luca [27] studied the fractional BVP

$$
\begin{cases}D_{0^{+}}^{\alpha} x(t)+\lambda f(t, x(t))=0, & 0<t<1, \\ x(0)=x^{\prime}(0)=\cdots=x^{(n-2)}(0)=0, & D_{0^{+}}^{\beta} x(1)=\sum_{i=1}^{m} \alpha_{i} D_{0^{+}}^{\gamma} x\left(\xi_{i}\right),\end{cases}
$$

where $\lambda>0,2 \leq n-1<\alpha \leq n, \alpha_{i} \geq 0,0<\xi_{1}<\xi_{2}<\cdots<\xi_{m}<1(i=1,2, \ldots, m), 1 \leq \beta \leq$ $n-2,0 \leq \gamma \leq \beta$, and $f(t, x)$ may be singular at $t=0,1$ and may change sign. In [28], for

(c) The Author(s) 2018. This article is distributed under the terms of the Creative Commons Attribution 4.0 International License (http://creativecommons.org/licenses/by/4.0/), which permits unrestricted use, distribution, and reproduction in any medium, provided you give appropriate credit to the original author(s) and the source, provide a link to the Creative Commons license, and indicate if changes were made. 
$\lambda=1$, the authors investigated the existence and multiplicity of positive solutions for BVP (1.2). In $[29,30]$, the authors discussed the following infinite-point BVP:

$$
\begin{cases}D_{0^{+}}^{\alpha} x(t)+f(t, x(t))=0, & 0<t<1, \\ x(0)=x^{\prime}(0)=\cdots=x^{(n-2)}(0)=0, & x^{(i)}(1)=\sum_{j=1}^{\infty} \alpha_{j} x\left(\xi_{j}\right),\end{cases}
$$

where $i \in\{1,2, \ldots, n-2\}$, and $\sum_{j=1}^{\infty} \alpha_{j} \xi_{j}^{\alpha-1}<(\alpha-1) \cdots(\alpha-i)$. The existence, uniqueness, and multiplicity of positive solutions for BVP (1.3) are established. Qiao and Zhou [31] discussed the singular BVP

$$
\begin{cases}D_{0^{+}}^{\alpha} x(t)+g(t) f(t, x(t))=0, & 0<t<1, \\ x(0)=x^{\prime}(0)=\cdots=x^{(n-2)}(0)=0, & D_{0^{+}}^{\beta} x(1)=\sum_{i=1}^{\infty} \alpha_{i} x\left(\xi_{i}\right),\end{cases}
$$

where $\beta \in[1, \alpha-1]$, and $\Gamma(\alpha)>\Gamma(\alpha-\beta) \sum_{i=1}^{\infty} \alpha_{i} \xi_{i}^{\alpha-1}$. For more results on the fractional infinite-point BVPs, see [24, 25, 32,33] and the references therein.

In the present paper, we investigate the existence of positive solutions for the singular fractional infinite-point BVP (1.1) using the fixed point index theory in cones. Note that $f(t, x)$ may be singular at $x=0$ and $g(t)$ may be singular at $t=0$ or $t=1$.

\section{Preliminaries and lemmas}

Definition 2.1 ([1-4]) The Riemann-Liouville fractional integral of order $\alpha>0$ of a function $h:(0,+\infty) \rightarrow \mathbb{R}$ is given by

$$
I_{0^{+}}^{\alpha} h(t)=\frac{1}{\Gamma(\alpha)} \int_{0}^{t}(t-s)^{\alpha-1} h(s) d s
$$

provided that the right-hand side is defined pointwise on $(0,+\infty)$.

Definition $2.2([1-4])$ The Riemann-Liouville fractional derivative of order $\alpha>0$ of a function $h:(0,+\infty) \rightarrow \mathbb{R}$ is given by

$$
D_{0^{+}}^{\alpha} h(t)=\frac{1}{\Gamma(n-\alpha)}\left(\frac{d}{d t}\right)^{n} \int_{0}^{t} \frac{h(s)}{(t-s)^{\alpha-n+1}} d s,
$$

where $n$ is the smallest integer not less than $\alpha$, provided that the right-hand side is defined pointwise on $(0,+\infty)$.

By arguments similar to those in [30,31], we have the following two lemmas.

Lemma 2.1 Given $y \in C(0,1) \cap L^{1}(0,1)$, the solution of the BVP

$$
\begin{cases}D_{0^{+}}^{\alpha} x(t)+y(t)=0, & t \in(0,1), \\ x(0)=x^{\prime}(0)=\cdots=x^{(n-2)}(0)=0, & D_{0^{+}}^{\beta} x(1)=\sum_{i=1}^{\infty} \alpha_{i} D_{0^{+}}^{\gamma} x\left(\xi_{i}\right),\end{cases}
$$

is

$$
x(t)=\int_{0}^{1} G(t, s) y(s) d s,
$$


where $G(t, s)$ is the Green's function given by

$$
G(t, s)=\frac{1}{\Gamma(\alpha) q(0)} \begin{cases}q(s)(1-s)^{\alpha-\beta-1} t^{\alpha-1}-q(0)(t-s)^{\alpha-1}, & 0 \leq s \leq t \leq 1, \\ q(s)(1-s)^{\alpha-\beta-1} t^{\alpha-1}, & 0 \leq t \leq s \leq 1\end{cases}
$$

and

$$
q(s)=\frac{1}{\Gamma(\alpha-\beta)}-\frac{1}{\Gamma(\alpha-\gamma)} \sum_{s \leq \xi_{i}} \alpha_{i}\left(\frac{\xi_{i}-s}{1-s}\right)^{\alpha-\gamma-1}(1-s)^{\beta-\gamma} .
$$

Lemma 2.2 The functions $q$ and $G$ given in Lemma 2.1 have the following properties:

(i) $q \in C([0,1],(0,+\infty))$ is nondecreasing;

(ii) $G(t, s) \in C([0,1] \times[0,1],[0,+\infty))$;

(iii) $p(t) G(1, s) \leq G(t, s) \leq G(1, s), t, s \in[0,1]$, where $p(t)=t^{\alpha-1}$.

Set $E=C[0,1]$ and $\|x\|=\sup _{t \in[0,1]}|x(t)|$. We define the cones

$$
P=\{x \in E: x(t) \geq 0, t \in[0,1]\} \quad \text { and } \quad K=\{x \in P: x(t) \geq p(t)\|x\|, t \in[0,1]\} .
$$

For $0<r<+\infty$, denote $K_{r}=\{x \in K:\|x\|<r\}, \partial K_{r}=\{x \in K:\|x\|=r\}$ and $\bar{K}_{r}=\{x \in K$ : $\|x\| \leq r\}$. Define the operators $A: \bar{K}_{R} \backslash K_{r} \rightarrow P$ and $L: E \rightarrow E$ by

$$
\begin{aligned}
& A x(t)=\lambda \int_{0}^{1} G(t, s) g(s) f(s, x(s)) d s, \quad t \in[0,1], \\
& L x(t)=\int_{0}^{1} G(t, s) g(s) x(s) d s, \quad t \in[0,1] .
\end{aligned}
$$

Clearly, $L: K \rightarrow K$ is a completely continuous linear operator. Moreover, if $x$ is a fixed point of $A$, then $x$ is a solution of BVP (1.1).

We further assume that:

$\left(H_{1}\right) g \in C((0,1),[0, \infty))$ and $0<\int_{0}^{1} G(1, s) g(s) d s<+\infty$.

$\left(H_{2}\right) f \in C([0,1] \times(0, \infty),[0, \infty))$, and for any $0<r<R<+\infty$,

$$
\lim _{m \rightarrow \infty} \sup _{u \in \bar{K}_{R} \backslash K_{r}} \int_{D(m)} g(s) f(s, x(s)) d s=0,
$$

where $D(m)=\left[0, \frac{1}{m}\right] \cup\left[\frac{m-1}{m}, 1\right]$.

We obtain the following lemma using proofs similar to those in $[34,35]$.

Lemma 2.3 Suppose that $\left(H_{1}\right)$ and $\left(H_{2}\right)$ hold. Then $A: \bar{K}_{R} \backslash K_{r} \rightarrow K$ is completely continuous.

By Lemma 2.2 we can show that the spectral radius $r(L)>0$; see, for example, Lemma 2.5 of [36]. Using the Krein-Rutman theorem (see Theorem 19.2 on p. 226 of [37]), we have the following result.

Lemma 2.4 Suppose that $\left(H_{1}\right)$ and $\left(H_{2}\right)$ are satisfied. Then the first eigenvalue of $L$ is $\lambda_{1}=$ $(r(L))^{-1}>0$, and there exists a positive eigenfunction $\varphi_{1}$ such that $\varphi_{1}=\lambda_{1} L \varphi_{1}$. 
The main tool in the paper is the following fixed point index theorem.

Lemma 2.5 ([38]) Let $K$ be a cone in a Banach space E, and let $T: \bar{K}_{r} \rightarrow K$ be a completely continuous operator.

(i) If there exists $u_{0} \in K \backslash\{\theta\}$ such that $u-T u \neq \mu u_{0}$ for any $u \in \partial K_{r}$ and $\mu \geq 0$, then $i\left(T, K_{r}, K\right)=0$.

(ii) If $T u \neq \mu u$ for any $u \in \partial K_{r}$ and $\mu \geq 1$, then $i\left(T, K_{r}, K\right)=1$.

\section{Main results}

Theorem 3.1 Suppose that $\left(H_{1}\right)$ and $\left(H_{2}\right)$ are satisfied. If

$$
0 \leq f^{\infty}:=\limsup _{x \rightarrow+\infty} \max _{t \in[0,1]} \frac{f(t, x)}{x}<\lambda_{1}<f_{0}:=\liminf _{x \rightarrow 0} \min _{t \in[0,1]} \frac{f(t, x)}{x} \leq+\infty
$$

then BVP (1.1) has at least one positive solution for any

$$
\lambda \in\left(\frac{\lambda_{1}}{f_{0}}, \frac{\lambda_{1}}{f^{\infty}}\right)
$$

Proof By (3.1) we have $f_{0}>\frac{\lambda_{1}}{\lambda}$, and there exists $r_{1}>0$ such that $f(t, x) \geq \frac{\lambda_{1}}{\lambda} x$ for $0<x \leq r_{1}$ and $0 \leq t \leq 1$. For any $x \in \partial K_{r_{1}}$, we obtain

$$
(A x)(t)=\lambda \int_{0}^{1} G(t, s) g(s) f(s, x(s)) d s \geq \lambda_{1}(L x)(t), \quad t \in[0,1]
$$

Suppose that $\varphi_{1}$ is the positive eigenfunction corresponding to $\lambda_{1}$ and that $A$ has no fixed points on $\partial K_{r_{1}}$. We claim that

$$
x-A x \neq \mu \varphi_{1}, \quad x \in \partial K_{r_{1}}, \mu \geq 0 .
$$

Otherwise, there would exist $x_{1} \in \partial K_{r_{1}}$ and $\mu_{1} \geq 0$ such that $x_{1}-A x_{1}=\mu_{1} \varphi_{1}$. Then $\mu_{1}>0$ and $x_{1}=A x_{1}+\mu_{1} \varphi_{1} \geq \mu_{1} \varphi_{1}$. Denote $\bar{\mu}=\sup \left\{\mu \mid x_{1} \geq \mu \varphi_{1}\right\}$. Then $\bar{\mu} \geq \mu_{1}, x_{1} \geq \bar{\mu} \varphi_{1}$, and $A x_{1} \geq \lambda_{1} \bar{\mu} L \varphi_{1}=\bar{\mu} \varphi_{1}$. Thus

$$
x_{1}=A x_{1}+\mu_{1} \varphi_{1} \geq \bar{\mu} \varphi_{1}+\mu_{1} \varphi_{1}=\left(\bar{\mu}+\mu_{1}\right) \varphi_{1},
$$

which contradicts to the definition of $\bar{\mu}$. It follows from (3.2) and Lemma 2.5(i) that

$$
i\left(A, K_{r_{1}}, K\right)=0 .
$$

On the other hand, by (3.1) we have $f^{\infty}<\frac{\lambda_{1}}{\lambda}$, and there exist $r_{2}>r_{1}$ and $0<\sigma<1$ such that $f(t, x) \leq \sigma \frac{\lambda_{1}}{\lambda} x$ for $x \geq r_{2}$ and $0 \leq t \leq 1$. We define $L_{1} u=\sigma \lambda_{1} L u$. Obviously, the linear operator $L_{1}: E \rightarrow E$ is bounded, and $L_{1}(K) \subset K$. From the definition of $\lambda_{1}$ and $0<\sigma<1$ it follows that

$$
\left(r\left(L_{1}\right)\right)^{-1}=\left(\sigma \lambda_{1}\right)^{-1}(r(L))^{-1}=\sigma^{-1}>1 .
$$


Choose $\varepsilon_{0}=\frac{1}{2}\left(1-r\left(L_{1}\right)\right)$. Then by Gelfand's formula there exists a natural number $N \geq 1$ such that $\left\|L_{1}^{k}\right\| \leq\left[r\left(L_{1}\right)+\varepsilon_{0}\right]^{k}$ for $k \geq N$. We now define

$$
\|x\|^{*}=\sum_{i=1}^{N}\left[r\left(L_{1}\right)+\varepsilon_{0}\right]^{N-i}\left\|L_{1}^{i-1} x\right\|, \quad x \in E,
$$

where $L_{1}^{0}=I$ is the identity operator. Since $L_{1}$ is linear, it is easy to verify that $\|x\|^{*}$ is a norm in $E$. Let $M_{0}=\sup _{x \in \partial K_{r_{2}}} \lambda \int_{0}^{1} G(1, s) g(s) f(s, x(s)) d s$. Then $M_{0}<+\infty$. We define $M_{0}^{*}=\left\|M_{0}\right\|^{*}$ and take $r_{3}>\max \left\{r_{2}, 2 M_{0}^{*} \varepsilon_{0}^{-1}\right\}$. Noting that $\|x\|^{*}>\left[r\left(L_{1}\right)+\varepsilon_{0}\right]^{N-1}\|x\|$, we can find $r_{4}>r_{3}$ large enough such that $\|x\| \geq r_{4}$ and thus $\|x\|^{*}>r_{3}$.

We next prove that

$$
A x \neq \mu x, \quad x \in \partial K_{r_{4}}, \mu \geq 1 .
$$

Arguing indirectly, we get that there exist $x_{2} \in \partial K_{r_{4}}$ and $\mu_{2} \geq 1$ such that $A x_{2}=\mu_{2} x_{2}$. We define $\widetilde{x}(t)=\min \left\{x_{2}(t), r_{2}\right\}$ for $t \in[0,1]$ and $H\left(x_{2}\right)=\left\{t \in[0,1]: x_{2}(t)>r_{2}\right\}$. It is easy to see that $\|\widetilde{x}\|=r_{2}$. We have $\widetilde{x} \in \partial K_{r_{2}}$ since $\widetilde{x}(t)=\min \left\{x_{2}(t), r_{2}\right\} \geq \min \left\{p(t) r_{4}, r_{2}\right\} \geq p(t) r_{2}$, $t \in[0,1]$. It follows that

$$
\begin{aligned}
\mu_{2} x_{2}(t) & =\left(A x_{2}\right)(t) \\
& =\lambda \int_{0}^{1} G(t, s) g(s) f\left(s, x_{2}(s)\right) d s \\
& \leq \lambda \int_{H\left(x_{2}\right)} G(t, s) g(s) f\left(s, x_{2}(s)\right) d s+\lambda \int_{[0,1] \backslash H\left(x_{2}\right)} G(1, s) g(s) f\left(s, x_{2}(s)\right) d s \\
& \leq \sigma \lambda_{1} \int_{0}^{1} G(t, s) g(s) x_{2}(s) d s+\lambda \int_{0}^{1} G(1, s) g(s) f(s, \widetilde{x}(s)) d s \\
& \leq\left(L_{1} x_{2}\right)(t)+M_{0}, \quad t \in[0,1] .
\end{aligned}
$$

Since $L_{1}(K) \subset K$, we have $0 \leq\left(L_{1}^{j}\left(A x_{2}\right)(t)\right) \leq\left(L_{1}^{j}\left(L_{1} x_{2}+M_{0}\right)(t)\right), j=0,1,2, \ldots, N-1$. Then $\left\|L_{1}^{j}\left(A x_{2}\right)\right\| \leq\left\|L_{1}^{j}\left(L_{1} x_{2}+M_{0}\right)\right\|, j=0,1,2, \ldots, N-1$, and hence

$$
\left\|A x_{2}\right\|^{*} \leq \sum_{i=1}^{N}\left[r\left(L_{1}\right)+\varepsilon_{0}\right]^{N-i}\left\|L_{1}^{i-1}\left(L_{1} x_{2}+M_{0}\right)\right\|=\left\|L_{1} x_{2}+M_{0}\right\|^{*} .
$$

Therefore we obtain

$$
\begin{aligned}
\mu_{2}\left\|x_{2}\right\|^{*} & =\left\|A x_{2}\right\|^{*} \\
& \leq\left\|L_{1} x_{2}\right\|^{*}+M_{0}^{*} \\
& =\sum_{i=1}^{N}\left[r\left(L_{1}\right)+\varepsilon_{0}\right]^{N-i}\left\|L_{1}^{i} x_{2}\right\|+M_{0}^{*} \\
& \leq\left[r\left(L_{1}\right)+\varepsilon_{0}\right] \sum_{i=1}^{N-1}\left[r\left(L_{1}\right)+\varepsilon_{0}\right]^{N-i-1}\left\|L_{1}^{i} x_{2}\right\|+\left[r\left(L_{1}\right)+\varepsilon_{0}\right]^{N}\left\|x_{2}\right\|+M_{0}^{*} \\
& =\left[r\left(L_{1}\right)+\varepsilon_{0}\right] \sum_{i=1}^{N}\left[r\left(L_{1}\right)+\varepsilon_{0}\right]^{N-i}\left\|L_{1}^{i-1} x_{2}\right\|+M_{0}^{*}
\end{aligned}
$$




$$
\begin{aligned}
& =\left[r\left(L_{1}\right)+\varepsilon_{0}\right]\left\|x_{2}\right\|^{*}+M_{0}^{*} \\
& \leq\left[r\left(L_{1}\right)+\varepsilon_{0}\right]\left\|x_{2}\right\|^{*}+\frac{\varepsilon_{0}}{2} r_{3} \\
& <\left[r\left(L_{1}\right)+\varepsilon_{0}\right]\left\|x_{2}\right\|^{*}+\frac{\varepsilon_{0}}{2}\left\|x_{2}\right\|^{*} \\
& =\left[\frac{1}{4} r\left(L_{1}\right)+\frac{3}{4}\right]\left\|x_{2}\right\|^{*} .
\end{aligned}
$$

Thus $\frac{1}{4} r\left(L_{1}\right)+\frac{3}{4} \geq 1$, that is, $r\left(L_{1}\right) \geq 1$, which contradicts (3.4). It follows from (3.5) and Lemma 2.5(ii) that

$$
i\left(A, K_{r_{4}}, K\right)=1 \text {. }
$$

By (3.3), (3.6), and the additivity of the fixed point index we have

$$
i\left(A, K_{r_{4}} \backslash \bar{K}_{r_{1}}, K\right)=i\left(A, K_{r_{4}}, K\right)-i\left(A, K_{r_{1}}, K\right)=1
$$

Therefore $A$ has at least one fixed point $x^{*} \in K_{r_{4}} \backslash \bar{K}_{r_{1}}$, which is a positive solution of BVP (1.1).

\section{An example}

Let $\alpha=\frac{7}{2}, \beta=\frac{3}{2}, \gamma=\frac{1}{2}, \alpha_{i}=\frac{2}{i^{2}}, \xi_{i}=1-\frac{1}{i+1}(i=1,2, \ldots), g(t)=\frac{1}{\sqrt[4]{t(1-t)}}, f(t, x)=\sqrt{2-t+|\ln x|}$. Consider the following fractional BVP:

$$
\begin{cases}D_{0^{+}}^{\frac{7}{2}} x(t)+\lambda \frac{1}{\sqrt[4]{t(1-t)}} \sqrt{2-t+|\ln x(t)|}=0, & t \in(0,1), \\ x(0)=x^{\prime}(0)=x^{\prime \prime}(0)=0, & D_{0^{+}}^{\frac{3}{2}} x(1)=\sum_{i=1}^{\infty} \frac{2}{i^{2}} D_{0^{+}}^{\frac{1}{2}} x\left(1-\frac{1}{i+1}\right) .\end{cases}
$$

Direct computation shows that $\Gamma(\alpha-\beta)=1, \Gamma(\alpha-\gamma)=2, \sum_{i=1}^{\infty} \alpha_{i} \xi_{i}^{\alpha-\gamma-1}=2\left(\frac{\pi^{2}}{6}-1\right)$, and $\frac{1}{\Gamma(\alpha-\beta)}-\frac{1}{\Gamma(\alpha-\gamma)} \sum_{i=1}^{\infty} \alpha_{i} \xi_{i}^{\alpha-\gamma-1} \approx 0.355>0$.

Let $K=\{x \in C[0,1]: x(t) \geq p(t)\|x\|, t \in[0,1]\}$, where $p(t)=t^{\frac{5}{2}}$. For $x \in \bar{K}_{R} \backslash K_{r}$, we obtain $|\ln x(t)| \leq|\ln r p(t)|+|\ln R|$. Due to $\int_{0}^{1}|\ln p(t)| d t=\frac{5}{2}$, we have $\lim _{m \rightarrow \infty} \int_{D(m)}|\ln p(t)| d t=0$. Since $0 \leq G(t, s) \leq G(1, s) \leq \frac{1}{\Gamma\left(\frac{7}{2}\right)\left(2-\frac{\pi^{2}}{6}\right)}$, it follows that $\int_{0}^{1} G(1, s) g(s) d s \leq \frac{1}{\Gamma\left(\frac{7}{2}\right)\left(2-\frac{\pi^{2}}{6}\right)} \times$ $\int_{0}^{1} g(s) d s=\frac{2\left[\Gamma\left(\frac{3}{4}\right)\right]^{2}}{\Gamma\left(\frac{7}{2}\right)\left(2-\frac{\pi^{2}}{6}\right) \sqrt{\pi}}$. For $x \in \bar{K}_{R} \backslash K_{r}$, we have

$$
\int_{0}^{1} f^{2}(s, x(s)) d s \leq \int_{0}^{1}(2-s+|\ln r|+|\ln R|+|\ln p(s)|) d s=4+|\ln r|+|\ln R| .
$$

Therefore

$$
\begin{aligned}
& \lim _{m \rightarrow \infty} \sup _{x \in \bar{K}_{R} \backslash K_{r}} \int_{D(m)} g(s) f(s, x(s)) d s \\
& \leq \lim _{m \rightarrow \infty} \sup _{x \in \bar{K}_{R} \backslash K_{r}}\left(\int_{D(m)} g^{2}(s) d s\right)^{\frac{1}{2}}\left(\int_{D(m)} f^{2}(s, x(s)) d s\right)^{\frac{1}{2}} \\
& \leq \lim _{m \rightarrow \infty} \sqrt{\pi}\left(\int_{D(m)}(2-s+|\ln r|+|\ln R|+|\ln p(s)|) d s\right)^{\frac{1}{2}}=0 .
\end{aligned}
$$


Direct computation yields $f^{\infty}=0$ and $f_{0}=+\infty$. Using Theorem 3.1, we can conclude that the BVP (4.1) has at least one positive solution for any $\lambda \in(0,+\infty)$.

\section{Conclusions}

We established the existence of positive solutions for the singular fractional differential equation infinite-point BVP (1.1) using the fixed point index theory in cones. Note that the nonlinearity may possess singularities, that is, $f(t, x)$ may have a singularity at $x=0$, and $g(t)$ may be singular at $t=0$ or $t=1$.

\section{Funding}

Supported financially by the National Natural Science Foundation of China (11501318, 11871302), the China Postdoctoral Science Foundation (2017M612230), and the Natural Science Foundation of Shandong Province of China (ZR2017MA036).

\section{Availability of data and materials}

Not applicable.

\section{Ethics approval and consent to participate}

Not applicable.

\section{Competing interests}

The authors declare that they have no competing interests.

\section{Consent for publication}

Not applicable.

\section{Authors' contributions}

The authors contributed equally to this paper. All authors read and approved the final manuscript.

\section{Author details}

${ }^{1}$ School of Mathematical Sciences, Qufu Normal University, Qufu, P.R. China. ${ }^{2}$ Weifang Engineer Vocational College, Shandong, P.R. China.

\section{Publisher's Note}

Springer Nature remains neutral with regard to jurisdictional claims in published maps and institutional affiliations.

Received: 22 July 2018 Accepted: 10 October 2018 Published online: 22 October 2018

\section{References}

1. Podlubny, I.: Fractional Differential Equations. Academic Press, New York (1999)

2. Kilbas, A.A., Srivastava, H.M., Trujillo, J.J.: Theory and Applications of Fractional Differential Equations. Elsevier, Amsterdam (2006)

3. Miller, K.S., Ross, B.: An Introduction to the Fractional Calculus and Fractional Differential Equations. Wiley, New York (1993)

4. Zhou, Y: Basic Theory of Fractional Differential Equations. World Scientific, Singapore (2014)

5. Goodrich, C.S.: Coercive nonlocal elements in fractional differential equations. Positivity 21, 377-394 (2017)

6. Shabibi, M., Postolache, M., Rezapour, Sh.: Positive solutions for a singular sum fractional differential system. Int. J. Anal. Appl. 13, 108-118 (2017)

7. Shabibi, M., Postolache, M., Rezapour, Sh., Vaezpour, S.M.: Investigation of a multi-singular pointwise defined fractional integro-differential equation. J. Math. Anal. 7, 61-77 (2016)

8. Zhang, X., Liu, L., Wu, Y.: The uniqueness of positive solution for a fractional order model of turbulent flow in a porous medium. Appl. Math. Lett. 37, 26-33 (2014)

9. Zhang, X., Mao, C., Liu, L., Wu, Y.: Exact iterative solution for an abstract fractional dynamic system model for bioprocess. Qual. Theory Dyn. Syst. 16, 205-222 (2017)

10. Zhang, X., Liu, L., Wu, Y., Wiwatanapataphee, B.: The spectral analysis for a singular fractional differential equation with a signed measure. Appl. Math. Comput. 257, 252-263 (2015)

11. Zhang, X., Liu, L., Wu, Y., Lu, Y.: The iterative solutions of nonlinear fractional differential equations. Appl. Math. Comput. 219, 4680-4691 (2013)

12. Cui, Y.: Uniqueness of solution for boundary value problems for fractional differential equations. Appl. Math. Lett. 51, 48-54 (2016)

13. Cui, Y., Ma, W., Sun, Q., Su, X.: New uniqueness results for boundary value problem of fractional differential equation. Nonlinear Anal., Model. Control 23, 31-39 (2018)

14. Yan, F., Zuo, M., Hao, X.: Positive solution for a fractional singular boundary value problem with $p$-Laplacian operator. Bound. Value Probl. 2018, Article ID 51 (2018)

15. Zou, Y., He, G.: On the uniqueness of solutions for a class of fractional differential equations. Appl. Math. Lett. 74 68-73 (2017) 
16. Hao, X.: Positive solution for singular fractional differential equations involving derivatives. Adv. Differ. Equ. 2016, Article ID 139 (2016)

17. Hao, X., Wang, H., Liu, L., Cui, Y.: Positive solutions for a system of nonlinear fractional nonlocal boundary value problems with parameters and p-Laplacian operator. Bound. Value Probl. 2017, Article ID 182 (2017)

18. Zuo, M., Hao, X., Liu, L., Cui, Y.: Existence results for impulsive fractional integro-differential equation of mixed type with constant coefficient and antiperiodic boundary conditions. Bound. Value Probl. 2017. Article ID 161 (2017)

19. Hao, X., Zuo, M., Liu, L.: Multiple positive solutions for a system of impulsive integral boundary value problems with sign-changing nonlinearities. Appl. Math. Lett. 82, 24-31 (2018)

20. Zhang, X., Zhong, Q.: Uniqueness of solution for higher-order fractional differential equations with conjugate type integral conditions. Fract. Calc. Appl. Anal. 20, 1471-1484 (2017)

21. Zhang, X., Zhong, Q.: Triple positive solutions for nonlocal fractional differential equations with singularities both on time and space variables. Appl. Math. Lett. 80, 12-19 (2018)

22. Hao, X., Sun, H., Liu, L.: Existence results for fractional integral boundary value problem involving fractional derivatives on an infinite interval. Math. Meth. Appl. Sci. (2018). https://doi.org/10.1002/mma.5210

23. Hao, X., Wang, H.: Positive solutions of semipositone singular fractional differential systems with a parameter and integral boundary conditions. Open Math. 16, 581-596 (2018)

24. Guo, L., Liu, L., Wu, Y.: Existence of positive solutions for singular fractional differential equations with infinite-point boundary conditions. Nonlinear Anal., Model. Control 21, 635-650 (2016)

25. Guo, L., Liu, L., Wu, Y.: Existence of positive solutions for singular higher-order fractional differential equations with infinite-point boundary conditions. Bound. Value Probl. 2016, Article ID 114 (2016)

26. Salen, H.A.H.: On the fractional order $m$-point boundary value problem in reflexive Banach spaces and weak topologies. J. Comput. Appl. Math. 224, 565-572 (2009)

27. Henderson, J., Luca, R.: Existence of positive solutions for a singular fractional boundary value problem. Nonlinear Anal., Model. Control 22, 99-114 (2017)

28. Pu, R., Zhang, X., Cui, Y., Li, P., Wang, W.: Positive solutions for singular semipositone fractional differential equation subject to multipoint boundary conditions. J. Funct. Spaces 2017, Article ID 5892616 (2017)

29. Zhai, C., Wang, L.: Some existence, uniqueness results on positive solutions for a fractional differential equation with infinite-point boundary conditions. Nonlinear Anal., Model. Control 22, 566-577 (2017)

30. Zhang, X.: Positive solutions for a class of singular fractional differential equations with infinite-point boundary value conditions. Appl. Math. Lett. 39, 22-27 (2015)

31. Qiao, Y., Zhou, Z.: Existence of positive solutions of singular fractional differential equations with infinite-point boundary conditions. Adv. Differ. Equ. 2017, Article ID 8 (2017)

32. Zhong, Q., Zhang, X.: Positive solution for higher-order singular infinite-point fractional differential equation with p-Laplacian. Adv. Differ. Equ. 2016, Article ID 11 (2016)

33. Li, B., Sun, S., Sun, Y.: Existence of solutions for fractional Langevin equation with infinite-point boundary conditions. J. Appl. Math. Comput. 53, 683-692 (2017)

34. Hao, X., Liu, L., Wu, Y., Sun, Q.: Positive solutions for nonlinear nth-order singular eigenvalue problem with nonlocal conditions. Nonlinear Anal. 73, 1653-1662 (2010)

35. Wang, Y., Liu, L., Wu, Y.: Positive solutions for a nonlocal fractional differential equation. Nonlinear Anal. 74, 3599-3605 (2011)

36. Webb, J.R.L., Lan, K.Q.: Eigenvalue criteria for existence of multiple positive solutions of nonlinear boundary value problems of local and nonlocal type. Topol. Methods Nonlinear Anal. 27, 91-115 (2006)

37. Deimling, K.: Nonlinear Functional Analysis. Spring, Berlin (1985)

38. Guo, D., Lakshmikantham, V.: Nonlinear Problems in Abstract Cones. Academic Press, San Diego (1988)

\section{Submit your manuscript to a SpringerOpen ${ }^{\circ}$ journal and benefit from:}

- Convenient online submission

- Rigorous peer review

- Open access: articles freely available online

- High visibility within the field

- Retaining the copyright to your article

Submit your next manuscript at $\gg$ springeropen.com 